\title{
Pathological Response after Neoadjuvant Chemotherapy and Trastuzomab for Locally Advanced Her 2 Positive Breast Cancer. Experience of a Single institute
}

\author{
E. Ibrahim, M. Rahal, H. Abdelkhalek, A. Arini, N. Al Naimy, A. Al Garni and A. AlFaraj \\ Medical Oncology, Surgical Oncology, Pathology and Radiology Departments King Fahad Specialist \\ Hospital, Dammam, Saudi-Arabia
}

Background: Primary systemic chemotherapy is an established standard of care for LABC. Pathologic complete response (pCR) and minimal residual cancer burden (pT1a) are predictors for better disease free and overall survival. This retrospective study evaluates the clinical activity of sequential Adriamycin and Cyclophosphamide (AC) followed by Docetaxel- Cisplatin (D-Cis) and Trastuzumab (T) in locally advanced HER-2 positive breast cancer.

Methods: Non pretreated patients with invasive breast cancer, HER2 positive (IHC 3+) or FISH positive, stage IIIA-IIIB, with a baseline Left ventricular ejection fraction (LVEF) $\geq 50 \%$ were eligible. The neoadjuvant regimen consisted of 4 cycles of AC $\left(60 \mathrm{mg} / 600 \mathrm{mg} / \mathrm{m}^{2}\right)$ followed by 4 cycles of D- Cis $\left(75 \mathrm{mg} / \mathrm{m}^{2}\right.$ each) before surgery. Trastuzumab (T) started with the first cycle of D-Cis at $8 \mathrm{mg} / \mathrm{Kg}$ as loading dose then $6 \mathrm{mg} / \mathrm{Kg}$ for 1 year. The cycles were given every 3 weeks.

Results: Thirty one patients were reviewed. Median age: 47 years (24-70), premenopausal: 17, median tumor size: $7 \mathrm{~cm}$ (3-15), stage IIB: 3, IIIA: 11, IIIB: 17, ER/PR negative: 19 while ER/PR positive: 10. The toxicity related to AC was mild; with febrile neutropenia (FN) occurring in 5 patients and all patients completed the planned 4 cycles; while the second sequence with D-Cis was completed in 23 patients only. F.N, renal impairment, and hypersensitivity reaction were seen in 3,4 and 1 patients respectively. Seven cases $(22.6 \%)$ developed grade III nausea and vomiting. An asymptomatic decline of 15 percent in the LVEF occurred in 3 patients. Clinical evaluation of response by RECIST criteria pre surgery: Overall Response (OR): 30/31 (96.8\%), Complete Response (CR) 9/31 (29\%), Partial Response (PR) 21/31 (67.7\%) and 1 (3.2\%) Progressive Disease (PD). The second sequence with D-Cis-T doubled the rate of clinical CR obtained with AC (from 29\% to 58.1\%). All patients had surgery (except one with disease progression). Pathological assessment revealed that $14(46.7 \%)$ patients had no residual invasive carcinoma in the breast (pCR); 4 (13.3\%) had residual invasive component less than $5 \mathrm{~mm}$ (pT1a) in the breast; $16(53.3 \%)$ had negative nodes; 10 patients $(33.3 \%)$ achieved complete pathological response in the breast and axilla. pCR in the breast and axilla occurred in $80 \%$ of patients ( $8 / 10$ patients) with PR negative tumors.

Conclusion: The combination of trastuzumab with neoadjuvant chemotherapy for HER2-positive breast cancer is active and well tolerated. The pathological complete response (pCR) was 33.3\%. Tumors which do not express progesterone receptor were associated with a high probability of pCR suggesting 2 different entities within the all HER-2 positive breast cancer.

Key words: neoadjuvant chemotherapy in breast cancer, trastuzumab and chemotherapy in breast cancer and pathologic complete response in HER 2 positive breast cancer.

Corresponding Author: Dr. Ehab Ibrahim E-mail: ehabhas@hotmail.com

\section{INTRODUCTION}

Breast cancer is the most common cancer among women worldwide. Every year more than one million new cases of breast cancer are diagnosed worldwide, and nearly 400,000 people will die of the disease annually ${ }^{1}$.

Breast cancer accounts for $19 \%$ of cancer cases diagnosed in Saudi Arabia according to the National Cancer Registry Incidence Report. Unfortunately, more than half of the cases are diagnosed in advanced stages ${ }^{2}$.

HER2-positive disease is diagnosed in up to $30 \%$ of all breast cancer cases. It demands special attention Kasr-El-Aini Journal Of Clinical Oncology And Nuclear Medicine because the tumours are typically fast-growing and there is a high likelihood of relapse ${ }^{3}$.

In general, neoadjuvant therapy is used in breast cancer patients for three major reasons: to improve the surgical options, to determine the response to neoadjuvant therapy, and to obtain long-term disease free survival ${ }^{4,5}$.

There is still no solid proof that the neoadjuvant chemotherapy has influenced the survival. A metaanalysis evaluated 9 randomized studies including a total of 3946 patients with breast cancer that 
comparing neoadjuvant to adjuvant chemotherapy had concluded that neoadjuvant chemotherapy was apparently equivalent to adjuvant therapy in terms of disease free survival and overall survival ${ }^{6}$. However, in two large studies by the National Surgical Adjuvant Breast and Bowel Project (NSABP) B-18 and B-27, subgroup analysis of patients experiencing a pathologic complete response (pCR) of invasive tumours showed a significantly prolonged overall survival ${ }^{7,8}$.

Most studies on the use of trastuzumab in a neoadjuvant setting have been single-arm studies without acomparator?

Buzdar et al. ${ }^{10}$. compared neoadjuvant 5-fluorouracil/ epirubicin/cyclophosphamide and paclitaxel chemotherapy with or without trastuzumab for the treatment of HER2-postive, operable breast cancer. The pathological complete remission $(\mathrm{pCR})$ rate, which was the primary endpoint, was strikingly superior in the chemotherapy plus trastuzumab arm, when compared with that in the chemotherapy-alone arm (65\% vs. $26 \%$, P $1 / 4$ 0.016). Chang et al. ${ }^{11}$. Conducted a randomized study comparing neoadjuvant docetaxel/carboplatin (DC) therapy with or without trastuzumab in women with locally advanced breast cancer. The pCR rate was $36.4 \%$ in the DC plus trastuzumab arm, in contrast to the rate of $9 \%$ in the DC-alone arm.

The current study aimed at evaluation of the pathological complete response (pCR), efficacy and safety of an anthracycline-taxane-cisplatinum-herceptin containing regimen as neoadjuvant chemotherapy in locally advanced HER 2 positive breast cancer.

\section{PATIENTS AND METHODS}

This retrospective study was conducted on thirty one patients with pathologically confirmed HER 2 positive locally advanced breast cancer (IIIA-IIIB) between June 2006 and March 2009 at King Fahad Specialist Hospital-Dammam, Saudi Arabia. Primary end point was pathologic complete response (pCR).

Initial evaluation, assessment of clinical and pathological response.

All patients underwent the followings: medical history, physical examination, tumor evaluation measurement and staging. Left ventricular ejection fraction (LVEF) evaluation by ECHO, complete blood cell count, serum chemistries, liver and renal function tests, bilateral mammography, Computed tomographic scan of chest, abdomen and pelvis and isotopic bone scan. Complete blood cell count, liver and renal profiles were measured before the administration of each chemotherapy cycle.
Clinical objective responses were evaluated according to Response Evaluation Criteria in Solid Tumor (RECIST) ${ }^{12}$. Complete response (CR) was defined as the disappearance of all known disease with no evidence of progressive disease; partial response (PR) was defined as a $>30 \%$ decrease in the sum of longest diameters of all target lesions with no appearance of new lesions. Progressive disease (PD) was defined as at least $20 \%$ increase in the sum of the longest diameters of all target lesions or the appearance of new lesions. Stable disease was defined neither as PR nor as PD.

\section{Pathological assessment}

Tumor samples were collected by needle core biopsy or surgical biopsy prior to systemic chemotherapy. ER, PR and HER-2/neu, were determined by standard immunohistochemistry (IHC). Tumors with less than $1 \%$ stained cells were considered to have Negative receptor status. HER-2/neu status was assessed by IHC and by fluorescence in situ hyperdization (FISH) confirmation if $2+$ immunohistochemistry staining was present. In our study, pathological response was considered complete (pCR) if no histological evidence of residual invasive or in situ tumor in all resected specimens of the breast and the axillary lymph nodes could be detected. Near complete pathological response (npCR) was considered if only occasionally scattered invasive tumor cells with a size of $5 \mathrm{~mm}$ or less in aggregates removed from the breast tissue at the time of definitive surgery.

\section{Treatment plan and follow up}

Neoadjuvant chemotherapy consisted of 4 cycles of AC $\left(60 \mathrm{mg} / \mathrm{m}^{2}, 600 \mathrm{mg} / \mathrm{m}^{2}\right)$ followed by 4 cycles consisting of D-Cis $\left(75 \mathrm{mg} / \mathrm{m}^{2}\right.$ each) every 3 weeks. Trastuzumab (T) started with the first cycle of Docetaxel-Cisplatin at $8 \mathrm{mg} /$ $\mathrm{Kg}$ as loading dose then $6 \mathrm{mg} / \mathrm{Kg}$ for 1year. The cycles were given every 3 weeks. All patients provided written consent. Clinical response, complete blood count and chemistry were assessed with every cycle. Echocardiography was done every 3 months to assess LT ventricular ejection fraction during Herceptin therapy. Surgery was performed on day 28 after completion of preoperative chemotherapy. Adjuvant radiotherapy and hormaonal treatment was administered after surgery whenever indicated. After completion of the treatment plan, follow up was carried out every three months. Toxicity was assessed using the Common Toxicity Criteria Version $2^{33}$.

\section{RESULTS}

Thirty one patients with stage IIIA-IIIB locally advanced breast cancer and HER 2 neo positive breast cancer were retrospectively analyzed during the period between June 2006 and March 2009. Patient's characteristics are shown in Table 1. Median age was 47 years with a range of (24$70)$. Seventeen patients $(54.8 \%)$ were premenopausal. The 
median tumor size was $7 \mathrm{~cm}$ with a range of $(3-15 \mathrm{~cm})$. Twenty five patients $(80.6 \%)$ were grade III and 25 patients $(80.6 \%)$ presented with palpable axillary lymph nodes. ER and PR negative phenotypes were seen in 19 patients (61.3\%) while ER and PR positive phenotypes were seen in 10 patients $(32.3 \%)$. Clinical response rate was $96.8 \%$ (30/31), of which clinical complete response rate was $29 \%$ (9/31). Progressive disease was detected in $3.2 \%$ of cases (one patient). All patients completed the anthracycline part with five episodes of febrile neutropenia for which G-CSF was added in subsequent cycles. Twenty three patients (74.2\%) completed all 4 cycles of Docetaxel-Cisplatin. Toxicity related to Docetaxel-Cisplatin was as follows: 3 patients $(9.7 \%)$ with febrile neutropenia, four patients $(12.9 \%)$ with renal impairment and 7 patients $(22.6 \%)$ with grade III nausea and vomiting. Base line Lt ventricular ejection fraction ranged from $50-71 \%$. None of the patients experienced cardiac toxicity during neoadjuvant part of Herceptin therapy. During adjuvant Herceptin therapy, three patients $(9.7 \%)$ dropped their ejection fraction below $50 \%$ and Herceptin therapy had to be interrupted and then resumed after normalization of their ejection fraction. Twenty eight patients underwent modified radical mastectomy and two underwent breast conserving surgery. One patient was not operated due to disease progression. The median yield of axillary lymph node dissection was 9 with a range between 5-20 lymph nodes.

Clinical and pathologic responses are shown in Table 2. At the end of chemotherapy eighteen patients (58.1\%) had complete clinical response. The second sequence with Docetaxel-Cisplatin-Trastuzumab doubled the rate of clinical CR obtained with AC. Pathological assessment revealed that $14(46.7 \%), 16(53.3 \%)$ and 10 $(33.3 \%)$ patients had pathologic complete response (no residual tumor cell in the breast or the axillary lymph nodes) in the breast, axilla and both respectively. Six patients $(20 \%)$ had only residual DCIS. Eight patients $(80 \%)$ who had pathologic complete response (pCR) were PR negative subsets.

Follow-up data are available for 31 patients. Local recurrence and distant failure were reported in 9 patients, of these, four occurred in patients with a pCR and five occurred in patients with residual invasive component.

At last follow up (Mach 2012) twenty one patients $(67.7 \%)$ were alive free of the disease Table 4.

Table 1: Patient's and tumor's characteristics.

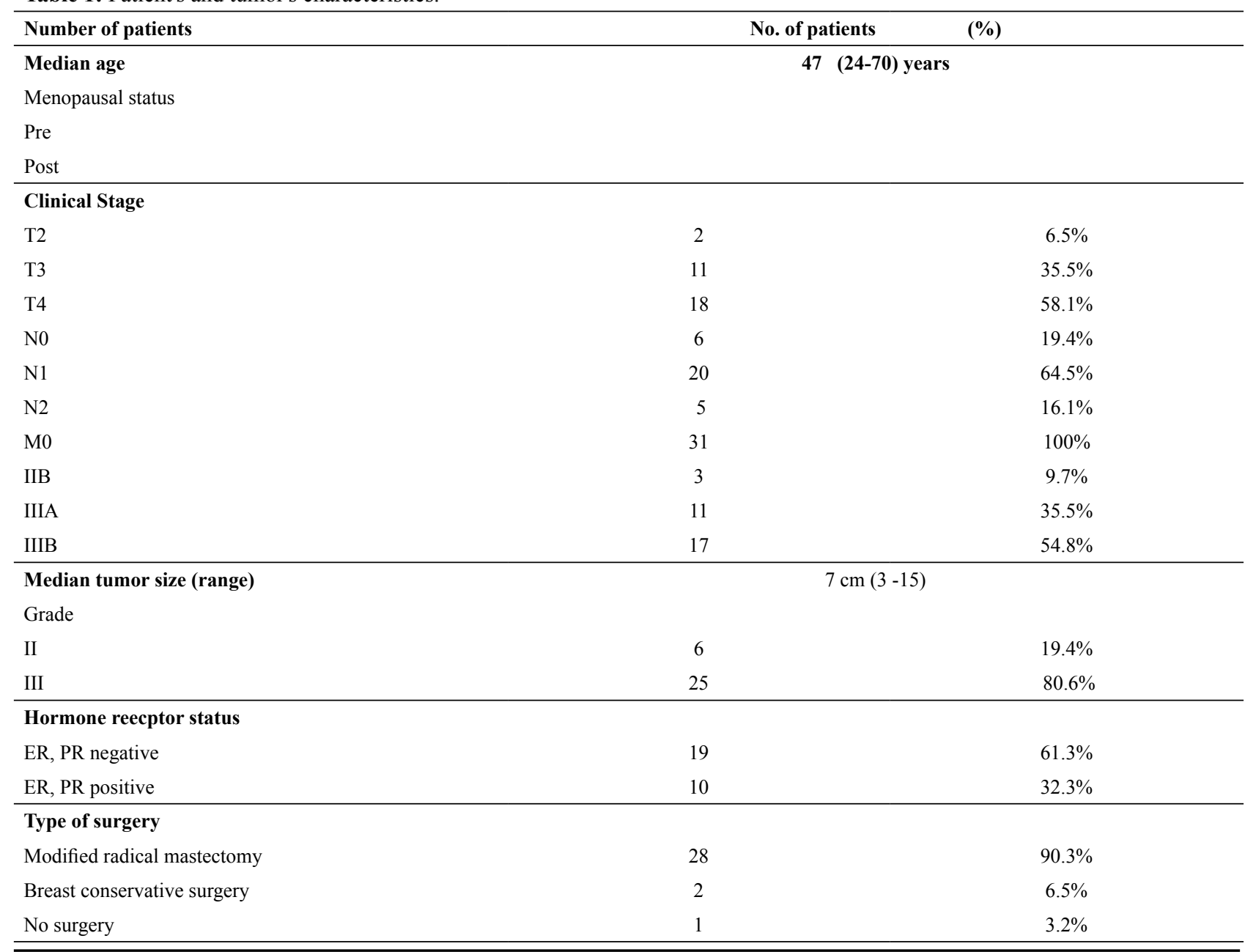


Table 2: Clinical and pathologic responses.

\begin{tabular}{lcc}
\hline Response & No. of patients & (\%) \\
\hline Clinical response after AC & 30 & $(96.8 \%)$ \\
Complete response (CR) & 9 & $(29 \%)$ \\
Partial response (PR) & 21 & $(67.7 \%)$ \\
\hline Clinical response after D-Cis-Herceptin & 30 & $(96.8 \%)$ \\
Complete response & 18 & $(58.1 \%)$ \\
Partial response & 12 & $(38.7 \%)$ \\
Pathologic complete response (Breast) & 14 & $(46.7 \%)$ \\
Pathologic complete response (Axilla) & 16 & $(53.3 \%)$ \\
Pathologic complete response & 10 & $(33.3 \%)$ \\
(Breast \& axilla) & & $(13.3 \%)$ \\
Residual invasive carcinoma less than $5 \mathrm{~mm}$ & 4 & $(20 \%)$ \\
Residual DCIS & 6 & \\
\hline
\end{tabular}

Table 3: Pathological response according to PR status.

PR -

$8 / 10$

Pathologic complete response

PR+

Table 2: Disease status at last follow up (March 2012)

\begin{tabular}{lcc}
\hline Status & Number & $\%$ \\
\hline Alive free of disease & 21 & 67.8 \\
Alive with disease & 3 & 9.7 \\
Died with disease & 7 & 22.6 \\
\hline
\end{tabular}

\section{DISCUSSION}

HER 2 overexpression occurs in approximately 20 to $25 \%$ of human breast cancers and is associated with an aggressive clinical phenotype that includes highgrade tumors, increased growth rates, early systemic metastases and decreased rates of disease-free and overall survival ${ }^{13,14}$.

Preclinical data indicate that this adverse clinical picture results from fundamental changes in the biologic features of breast-cancer cells containing the alteration, including increased proliferation, suppression of apoptosis, increased motility, greater invasive and metastatic potential, accelerated angiogenesis, and steroid hormone independence ${ }^{15-17}$.

Many studies had shown that these HER2-mediated adverse characteristics were reversed by the use of monoclonal antibodies directed against the tyrosine kinase receptor ${ }^{18-20}$ and these data led to phase 1 testing of a murine anti-HER 2 monoclonal antibody ${ }^{21}$. Preliminary efficacy and safety data prompted the development of a humanized monoclonal antibody to produce trastuzumab ${ }^{22}$. Subsequently, in a large, randomized study, the addition of trastuzumab to chemotherapy yielded significant improvements in rates of objective response, response duration, and time to disease progression $(56 \%$, $58 \%$, and $65 \%$ improvement, respectively), as well as a $30 \%$ improvement in the rate of overall survival among patients with first-line metastatic disease. A significant side effect was an increase by a factor of 4 in the rate of cardiac dysfunction, including congestive heart failure, especially when trastuzumab was used in combination with anthracycline-based regimens ${ }^{23}$.

Pre-operative systemic chemotherapy (PST) is the treatment of choice for women with locally advanced breast cancer ${ }^{6}$. It aims to reduce the tumor size and may allow for breast conserving.

surgery. Tumor response can be assessed clinically and pathologically and is valuable for testing new therapies. Pathological complete response (pCR) 


Vol. 9 | No. 1-2 $2013 \quad$ E. Ibrahim et al.

also provides a surrogate for disease-free and overall survival $^{24}$. The rationale for integrating trastuzumab into PST for HER2-positive breast cancer is based on the association between HER2 overexpression and poor prognosis ${ }^{25}$. Trastuzumab binds selectively to the HER2 receptor, provides significant clinical benefits including a survival advantage when combined with standard chemotherapy in HER2-positive metastatic breast cancer $^{26}$. Furthermore, when combined with docetaxel in patients with HER2-positive breast cancer, trastuzumab significantly improved all efficacy parameters, including overall survival, compared with docetaxel alone ${ }^{23}$.

In our study Trastuzumab plus neoadjuvant chemotherapy showed favorable safety and tolerability. No patients experienced symptomatic cardiac dysfunction. Only 3 patients had to interrupt treatment for a transient, asymptomatic decrease in LVEF and none of them had known risk factors for the cardiac dysfunction. The LVEF normalized 1 month after treatment was discontinued.

The overall clinical response rates observed in the current study appear very promising $(96.8 \%)$, which is comparable with previously reported other studies using trastuzumab-containing combination chemotherapy regimens. The Neoadjuvant Herceptin (NOAH) trial reported an overall response rate of $89 \%{ }^{27}$. In the current study the second sequence of chemotherapy with Docetaxel-Cisplatin-Trastuzumab almost doubled the rate of clinical complete response obtained with Adriamycin-Cyclophosphamide.

In our study, the pCR rate in both breast and axilla was $33.3 \%$ using the protocol predefined definition (excluding patients with remaining ductal carcinoma in situ) and 53.3\% using the now widely used definition of no invasive residual tumor in the breast and lymph nodes. Other trials reported an incidence of pCR of $60 \%, 43 \%$ and $31.7 \%$ respectively ${ }^{28-30}$.

In the GeparQuattro and TECHNO trials the authors reported an incidence of $31.7 \%$ and $39 \%$, respectively for $\mathrm{pCR}$ in patients who received Trastuzumab with neoadjuvant chemotherapy ${ }^{31,32}$.

In our study, despite the high pCR ratedid not result in a higher rate of breast-conserving surgery and this may be attributed to high percentage of patients who presented initially with T4 stage $(54.8 \%)$. Similar findings were reported in the GeparQuattro study ${ }^{31}$.

\section{CONCLUSIONS}

We have shown that the combination of trastuzumab with Adriamycin-Cyclophosphamide and Docetaxel-
Cisplatin as neoadjuvant treatment of HER2-positive locally advanced breast cancer is active and well tolerated. The pathological complete response rate (pCR) in patients with HER 2 positive locally advanced breast cancer was $33.3 \%$. As the sample size for this study was small, further assessment in a large trial is warranted.

\section{REFERENCES}

1. Ferlay J. GLOBOCAN 2002. Cancer Incidence, mortality and prevalence worldwide. IARC cancer base No.5, Version 2 Lyon: IARCPress; 2004.

2. Omalkhair A, Al Tahan F, Issa M, Jazieh A. The outcome of a public outreach screening program for breast cancer among Saudi women. ASCO, Breast Cancer Symposium 2007:Abstract No. 49.

3. Harries M, Smith I. The development and clinical use of trastuzumab (Herceptin). Endocr.Relat.Cancer 2002;9(2):75-85.

4. Kaufmann M, Hortobagyi GN, Goldhirsch A, Scholl S, Makris A, Valagussa $\mathrm{P}$, et al. Recommendations from an international expert panel on the use of neoadjuvant (primary) systemic treatment of operable breast cancer: An update. J.Clin.Oncol. 2006;24(12):1940-9.

5. Steger GG, Galid A, Gnant M, Mlineritsch B, Lang A, Tausch C, et al. Pathologic complete response with six compared with three cycles of neoadjuvant epirubicin plus docetaxel and granulocyte colony-stimulating factor in operable breast cancer: Results of ABCSG-14. J.Clin. Oncol. 2007;25(15):2012-8.

6. Mauri D, Pavlidis N, Ioannidis JP. Neoadjuvant versus adjuvant systemic treatment in breast cancer: A metaanalysis. J.Natl.Cancer Inst. 2005;97(3):188-94.

7. Fisher B, Bryant J, Wolmark N, Mamounas E, Brown A, Fisher ER, et al. Effect of preoperative chemotherapy on the outcome of women with operable breast cancer. J.Clin. Oncol. 1998;16(8):2672-85.

8. Bear HD, Anderson S, Smith RE, Geyer CEJ, Mamounas EP, Fisher B, et al. Sequential preoperative or postoperative docetaxel added to preoperative doxorubicin plus cyclophosphamide for operable breast cancer:National Surgical Adjuvant Breast and Bowel Project Protocol B-27. J.Clin.Oncol. 2006;24(13):2019-27.

9. Lazaridis G, Pentheroudakis G, Pavlidis N. Integrating trastuzumab in the neoadjuvant treatment of primary breast cancer: Accumulating evidence of efficacy, synergy and safety. Crit.Rev.Oncol.Hematol. 2008;66(1):31-41.

10. Buzdar AU, Ibrahim NK, Francis D, Booser DJ, Thomas ES, Theriault RL, et al. Significantly higher pathologic complete Remission Rate After Neoadjuvant Therapy With Trastuzumab, Paclitaxel and epirubicin chemotherapy: Results of a randomized trial in human epidermal growth factor receptor 2-positive operable breast cancer. J.Clin.Oncol. 2005;23(16):3676-85. 
11. Chang HR, Slamon D, Prati R, Glaspy J, Pegram MD, Kass FC, et al. A phase II study of neoadjuvant docetaxel/ carboplatin with or without trastuzumab in locally advanced breast cancer: Response and cardiotoxicity. J.Clin.Oncol. 2006;24(18 Suppl):S10515.

12. Therasse P, Arbuck SG, Eisenhauer EA, Wanders J, Kaplan RS, Rubinstein L, et al. New guidelines to evaluate the response to treatment in solid tumors. European Organization for Research and Treatment of Cancer, National Cancer Institute of the United States, National Cancer Institute of Canada. J.Natl.Cancer Inst. 2000;92(3):205-16.

13. Slamon DJ, Clark GM, Wong SG, Levin WJ, Ullrich A, McGuire WL. Human breast cancer: Correlation of relapse and survival with amplification of the HER-2/ neu oncogene. Science 1987;235(4785):177-82.

14. Slamon DJ, Godolphin W, Jones LA, Holt JA, Wong SG, Keith DE, et al. Studies of the HER-2/neu proto-oncogene in human breast and ovarian cancer. Science 1989;244(4905):707-12.

15. Arboleda MJ, Lyons JF, Kabbinavar FF, Bray MR, Snow BE, Ayala R, et al. Overexpression of AKT2/protein kinase Bbeta leads to up-regulation of betal integrins, increased invasion, and metastasis of human breast and ovarian cancer cells. Cancer Res. 2003;63(1):196-206.

16. Benz CC, Scott GK, Sarup JC, Johnson RM, Tripathy D, Coronado E, et al. Estrogen-dependent, tamoxifen-resistant tumorigenic growth of MCF-7 cells transfected with HER2/neu. Breast Cancer Res.Treat. 1992;24(2):85-95.

17. De Luca A, Carotenuto A, Rachiglio A, Gallo M, Maiello MR, Aldinucci D, et al. The role of the EGFR signaling in tumor microenvironment. J.Cell.Physiol. 2008;214(3):559-67.

18. Hudziak RM, Lewis GD, Winget M, Fendly BM, Shepard HM, Ullrich A. p185HER2 monoclonal antibody has antiproliferative effects in vitro and sensitizes human breast tumor cells to tumor necrosis factor. Mol.Cell.Biol. 1989;9(3):1165-72.

19. Hancock MC, Langton $\mathrm{BC}$, Chan $\mathrm{T}$, Toy $\mathrm{P}$, Monahan JJ, Mischak RP, et al. A monoclonal antibody against the c-erbB-2 protein enhances the cytotoxicity of cis-diamminedichloroplatinum against human breast and ovarian tumor cell lines. Cancer Res. 1991;51(17):4575-80.

20. Pegram MD, Slamon DJ. Combination therapy with trastuzumab (Herceptin) and cisplatin for chemoresistant metastatic breast cancer: evidence for receptor-enhanced chemosensitivity. Semin.Oncol. 1999;26(4 Suppl 12):89-95.

21. Slamon DJ. Final Study Report (FSR) for H0407g: A Phase 1 safety and tolerance study of intravenous anti-p185 HER2 humanized monoclonal antibody (rhu MAb HER2) in patients with HER2 overexpressing tumors. 1996. U.S.Food and Drug Administration.
22. Carter P, Presta L, Gorman CM, Ridgway JB, Henner D, Wong WL, et al. Humanization of an antip185HER2 antibody for human cancer therapy. Proc.Natl. Acad.Sci.U.S.A. 1992;89(10):4285-9.

23. Slamon DJ, Leyland-Jones B, Shak S, Fuchs H, Paton V, Bajamonde A, et al. Use of chemotherapy plus a monoclonal antibody against HER2 for metastatic breast cancer that overexpresses HER2. N.Engl.J.Med. 2001;344(11):783-92.

24. Kurosumi M. Significance of histopathological evaluation in primary therapy for breast cancer-recent trends in primary modality with pathological complete response (pCR) as endpoint. Breast Cancer 2004;11(2):139-47.

25. King $\mathrm{CR}$, Kraus $\mathrm{MH}$, Aaronson SA. Amplification of a novel v-erbB-related gene in a human mammary carcinoma. Science 1985;229(4717):974-6.

26. Marty M, Cognetti F, Maraninchi D, Snyder R, Mauriac L, Tubiana-Hulin M, et al. Randomized phase II trial of the efficacy and safety of trastuzumab combined with docetaxel in patients with human epidermal growth factor receptor 2-positive metastatic breast cancer administered as first-line treatment: The M77001 study group. J.Clin.Oncol. 2005;23(19):4265-74.

27. Gianni L, Eiermann W, Semiglazov V, Manikhas G, Lluch A, Tjulandin S, et al. Neoadjuvant trastuzumab in patients with HER2-positive locally advanced breast cancer: Primary efficacy analysis of the NOAH trial. Cancer Res. 2009;69(2 Suppl 1):Abstract 31.

28. Buzdar AU, Valero V, Ibrahim NK, Francis D, Broglio KR, Theriault RL, et al. Neoadjuvant therapy with paclitaxel followed by 5-fluorouracil, epirubicin, and cyclophosphamide chemotherapy and concurrent trastuzumab in human epidermal growth factor receptor 2-positive operable breast cancer: An update of the initial randomized study population and data of additional patients treated with the same regimen. Clin.Cancer Res. 2007;13(1):228-33.

29. Von Minckwitz G, Rezai M, Loibl S, Fasching PA, Juober J, Tesch $\mathrm{H}$, et al. Effect of trastuzumab on pathologic complete response rate of neoadjuvant ECdocetaxel treatment in Her2-overexpressing breast cancer: Results of the phase III GeparQuattro study. Presented at the ASCO Breast Cancer 2008: Abstract 226.

30. Gianni L, Eiermann W, Pusztai L, Semiglazov VF, Hoegel B, Koehler A, et al. Biomarkers as potential predictors of pathologic complete response (pCR) in the NOAH trial of neoadjuvant trastuzumab in patients (pts) with HER2-positive locally advanced breast cancer (LABC). J.Clin.Oncol.(Meeting Abstracts) 2008;26(15 Suppl):504.

31. Untch M, Rezai M, Loibl S, Fasching PA, Huober J, Tesch $\mathrm{H}$, et al. Neoadjuvant treatment with trastuzumab in HER2-positive breast cancer: results from the GeparQuattro study. J.Clin.Oncol. 2010;28(12):2024-31. 
32. Untch M, Fasching PA, Konecny GE, Hasmüller S, Lebeau A, Kreienberg R, et al. Pathologic complete response after neoadjuvant chemotherapy plus trastuzumab predicts favorable survival in human epidermal growth factor receptor 2-overexpressing breast cancer: Results from the TECHNO trial of the AGO and GBG study groups. J.Clin.Oncol. 2011;29(25):3351-7.
33. Therasse P, Arbuck SG, Eisenhauer EA, Wanders J, Kaplan RS, Rubinstein L, et al. New guidelines to evaluate the response to treatment in solid tumors. European Organization for Research and Treatment of Cancer, National Cancer Institute of the United States, National Cancer Institute of Canada. J.Natl.Cancer Inst. 2000;92(3):205-16. 